declared, Charles Masson: None declared, Divi Cornec: None declared, JeanJacques Dubost: None declared, Laurent Marguerie: None declared, Sebastien Ottaviani: None declared, Franck Grados: None declared, Rakiba Belkhir: None declared, olivier fain: None declared, Philippe Goupille Grant/research support from: AbbVie, Amgen, Biogen, BMS, Celgene, Chugai, Lilly, Janssen, Medac, MSD France, Nordic Pharma, Novartis, Pfizer, Sanofi and UCB, Consultant of: AbbVie, Amgen, Biogen, BMS, Celgene, Chugai, Lilly, Janssen, Medac, MSD France, Nordic Pharma, Novartis, Pfizer, Sanofi and UCB, Speakers bureau: AbbVie, Amgen, Biogen, BMS, Celgene, Chugai, Lilly, Janssen, Medac, MSD France, Nordic Pharma, Novartis, Pfizer, Sanofi and UCB, Christelle Sordet: None declared, Bruno Fautrel Grant/research support from: AbbVie, Lilly, MSD, Pfizer, Consultant of: AbbVie, Biogen, BMS, Boehringer Ingelheim, Celgene, Lilly, Janssen, Medac MSD France, Nordic Pharma, Novartis, Pfizer, Roche, Sanofi Aventis, SOBI and UCB, Peggy Philippe: None declared, Muriel PIPERNO: None declared, Bernard Combe Grant/research support from: Novartis, Pfizer, RocheChugai, Consultant of: AbbVie; Gilead Sciences, Inc.; Janssen; Eli Lilly and Company; Pfizer; Roche-Chugai; Sanofi, Speakers bureau: Bristol-Myers Squibb; Gilead Sciences, Inc.; Eli Lilly and Company; Merck Sharp \& Dohme; Pfizer; Roche-Chugai; UCB, Olivier Lambotte Consultant of: BMS France, MSD, Astra Zeneca, Incyte, Christophe Richez Consultant of: Abbvie, Amgen, Mylan, Pfizer, Sandoz and UCB., Jérémie SELLAM: None declared, Thomas Sene: None declared, Guillaume Denis: None declared, Thierry Lequerre: None declared, Xavier Mariette Consultant of: BMS, Gilead, Medimmune, Novartis, Pfizer, Servier, UCB, Gaetane Nocturne: None declared DOI: 10.1136/annrheumdis-2020-eular.3930

\section{OP0126 \\ IS IMMUNE CHECKPOINT INHIBITORS THERAPIES SAFE AND EFFECTIVE FOR PATIENTS WITH CANCER AND PREEXISTING AUTOIMMUNE DISEASE?}

W. Xie ${ }^{1}$, H. Huang ${ }^{1}$, Z. Zhang ${ }^{1}{ }^{1}$ Peking University First Hospital, Department of Rheumatology and Clinical Immunology, Beijing, China

Background: Immune checkpoints inhibitors (ICls) are associated with frequent immune-related adverse events (irAEs). Most patients with preexisting autoimmune disease (PAD) have been universally excluded from clinical trials and ICls are not recommended for patients with cancer and PAD due to the unknown safety. In this study, we aim to evaluate the safety and efficacy of ICls in patients with PAD and cancer.

Objectives: Systematic searches were performed of PubMed, EMBASE, and the Cochrane library from inception through September 2019 for observational studies reporting safety and efficacy data among ICls-treated patients with cancer and PAD. A random effects meta-analysis was performed to calculate pooled incidence rates of PAD flare, irAEs and response.

Methods: Systematical search of PubMed, EMBASE and Cochrane Library plus a hand search of conference proceedings were performed for observational studies that reported cancer incidence in patients with RA treated with biologics or tofacitinib with active comparator of conventional DMARDs (csDMARDs) or TNFi. The pooled relative risk $(\mathrm{RR})$ and $95 \%$ confidence interval $(\mathrm{Cl})$ were calculated with fix-effects or random-effects model.

Results: A total of $619 \mathrm{ICl}$-treated patients with PAD in 14 publications were finally identified. In the random effects meta-analysis, pooled incidence of PAD flares, de novo irAEs or both of any grade was $60 \%$ (95\% Cl 52\%-68\%). Viewed separately, there were 219 and 206 patients experiencing PAD exacerbation and de novo irAEs of any grade, yielding a pooled incidence of $35 \%(95 \% \mathrm{Cl} 29 \%$ $41 \%)$ and $33 \%(95 \% \mathrm{Cl} 24 \%-42 \%)$ respectively. Of these, most of flare and de novo irAEs were graded as mild (grade 1-2) (pooled proportion: $82 \%, 95 \% \mathrm{Cl}$ $72 \%-91 \% ; 65 \%, 95 \% \mathrm{Cl} 54 \%-76 \%$, respectively). Rheumatoid arthritis was associated with a trend toward higher flare occurrence compared with another individual PADs ( $R R=1.25-1.88)$. With respect to efficacy, 136 patients showed complete or partial response, corresponding to a pooled response rates of $30 \%(95 \% \mathrm{Cl}$ $22 \%-39 \%$ ). There were no statistical differences between patients with and without immunosuppressive therapy at $\mathrm{ICl}$ start regarding flare (RR: $1.08,95 \% \mathrm{Cl}$ $0.72-1.62)$, but a trend towards lower response rates was observed in patients with baseline immunosuppressants (RR: 0.58, 95\% Cl 0.26-1.33)

Conclusion: Immune toxicities are frequent in ICl-treated patients with PAD but often mild and manageable without discontinuing therapy. Rheumatoid arthritis is associated with a trend toward more flares. $\mathrm{ICI}$ treatment are effective and not absolute contraindication in PAD patients, but close monitoring and multidisciplinary collaboration should be contemplated, especially for those concomitantly receiving immunosuppressant or having rheumatoid arthritis.

References:

[1] Khan SA, Pruitt SL, Xuan L et al. Prevalence of autoimmune disease among patients with lung cancer: Implications for immunotherapy treatment options. JAMA Oncol 2016;2:1507-1508.
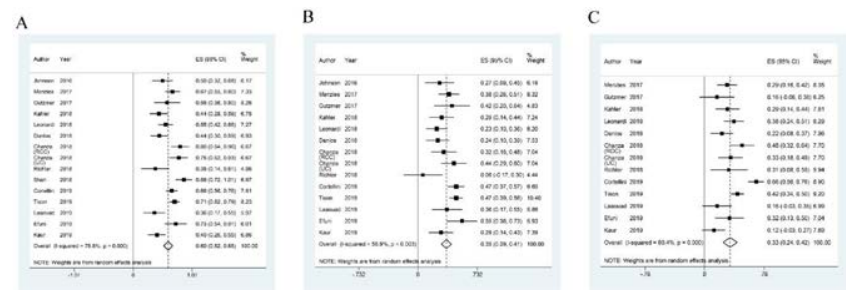

Figure 1. Pooled effect estimates in ICl-treated patient with PAD (A) PAD flare, de novo irAEs or both; (B) PAD flare; (C) de novo irAEs. ICl: Immune checkpoint inhibitors; PAD: Preexisting autoimmune disease; irAEs: Immune-related adverse events.

Disclosure of Interests: None declared

DOI: 10.1136/annrheumdis-2020-eular.5054

\section{Immunity in rheumatic disease}

\section{OP0127 CONTRIBUTES TO MATURATION AND FUNCTION OF THE INTERFERON-GAMMA PRODUCING T CELLS IN RHEUMATOID ARTHRITIS}

M. Erlandsson ${ }^{1,2}$, K. M. Andersson ${ }^{1}$, N. Nair ${ }^{3}$, A. Damdimopoulos ${ }^{4}$, S. Töyrä Silfverswärd ${ }^{1}$, R. Pullerits ${ }^{1,2}$, A. Barton ${ }^{3}$, M. I. Bokarewa ${ }^{1,2} .{ }^{1}$ Gothenburg University, Department of Rheumatology, Göteborg, Sweden; ${ }^{2}$ Sahlgrenska University Hospital, Rheumatology Clinic, Göteborg, Sweden; ${ }^{3}$ University of Manchester, Division of Musculoskeletal \& Dermatological Sciences, Manchester, United Kingdom; ${ }^{4}$ Karolinska Institute, H2 Department of Biosciences and Nutrition, Stockholm, Sweden

Background: Interferon gamma (IFNg) signalling and downstream effects make important contribution in pathogenesis of rheumatoid arthritis (RA). Here, we propose a mechanism by which oncoprotein survivin participates in development of IFN-dependent repertoire of T cells in RA patients.

Objectives: We study the role of survivin in the phenotype of CD4 T cells of RA patients.

Methods: CD4 cells of RA patients and healthy controls were purified from blood, activated and subjected to RNAseq, ChIPseq with antibodies to survivin (BIRC5) was performed on CD4+ cells. Histone H3 ChIPseq was performed using antibodies to $\mathrm{H} 3 \mathrm{~K} 27 \mathrm{ac}, \mathrm{H} 3 \mathrm{~K} 27 \mathrm{me} 3$ and $\mathrm{H} 3 \mathrm{~K} 4 \mathrm{me} 3$. Statistical analysis was performed In R-studio using the Bioconductor package DESeq2, clustering using Spearman and Ward.D2.

Results: Unsupervised clustering of CD4 samples by expression of 48 core Th cell markers identified subsets of $C D 28^{\text {hi }} C D 27^{\text {hi }} I F N^{\text {neg }}$ central memory cells $(\mathrm{Tcm}), \mathrm{CD}_{28}{ }^{\mathrm{lo}} \mathrm{CD} 27^{\mathrm{lo}} \mathrm{IFN}{ }^{\mathrm{lo}}$ effector memory cells (Tem) and CD28 ${ }^{\text {null }} \mathrm{CD} 27^{\text {null }} \mathrm{IF}$ $\mathrm{N}^{\text {hi }}$ terminal effector cells (Tte). Tte cells showed classical features of Th1 cells including high levels of TBX21, TNFa and IL32 and signs of activation in IFN signalling machinery. Interestingly, they combined the features of peripheral Tregs CD25 ${ }^{\text {hi }}$ Foxp $3^{\text {hi }}$ IKZF2 ${ }^{\text {neg }}$ and IL10 producing cells together with type 1 regulatory cells, which rely on transcription factors BATF and IRF1 for the differentiation and produced high amounts of perforin and granzyme B. Importantly, Tte CD4 cells had also high transcription of BIRC5 $(p=1.15 e-18)$.

To study if BIRC5 is a part of IFN signalling, CD4 cells were cultured with survivin inhibitor YM155 and activated with IFNg. RNAseq analysis revealed 2033 ( $F C<2.0, n=336$ ) differentially expressed genes in the IFN stimulated cultures. Interestingly, a substantial number of these IFN-dependent genes was significantly reduced in the survivin-deficient cultures and included among others CD28, FoxP3, IKZF2, ICOS, BATF, PRDM1, CXCR3, IRF4 and IRF8. Analysis of the peak sequences identified enrichment for composite motifs for IRFs (ETS:IRF, p1.0e-124; bZIP:IRF, $p=1.0 e-640$ ), indicating that survivin is important for IFNg signalling. Numerically, the peaks containing ETS:IRF motifs were most prevalent and identified in total within $49.7 \%$ of all survivin-ChIP peaks. Frequent was co-localisation of the IRF:bZIP and IRF:ETS motifs within the survivin peaks. Among the IRF motifs dominated those suitable for IRF1 $(p=1,0 e-127)$ and IRF8 $(p=1,0 e-84)$. However, the DNA binding motifs of these two are alike.

Encouraged by the survivin ChIPseq results, we wanted to know its relation to histone marks. We observed that $50 \%$ of survivin peaks containing both IRF:bZIP and IRF:ETS motifs are co-localized with the H3K27ac marks. In total, 16 of 48 core Th cell markers used for patients clustering were identified by survivin ChIPseq, co-localized with IRF composite motifs and histone marks. They were also dependent of survivin for expression. 